\title{
CD9 modulates proliferation of human glioblastoma cells via epidermal growth factor receptor signaling
}

\author{
GONG-PING WANG ${ }^{1}$ and XIAO-FANG HAN ${ }^{2}$ \\ ${ }^{1}$ First Department of Neurosurgery; ${ }^{2}$ Department of Medical Teaching, Xianyang Hospital of Yanan University, \\ Central Hospital of 20th Bureau of China Railway Group, Xianyang, Shaanxi 712000, P.R. China
}

Received March 8, 2014; Accepted January 2, 2015

DOI: $10.3892 / \mathrm{mmr} .2015 .3466$

\begin{abstract}
The tetraspanin CD9 has previously been shown to be involved in various cellular activities, including proliferation and migration. In addition, CD9 has been shown to be associated with epidermal growth factor receptor (EGFR). A common characteristic of glioblastoma multiforme histology is EGFR amplification, which affects signal transduction processes. The anti-proliferative effects of CD9 have been linked to EGFR signaling pathways, including phosphorylation of phosphoinositide-3-kinase (PI3K)/Akt and activation of mitogen-activated protein kinase (MAPK)/extracellular signal-regulated protein kinase (Erk). The present study demonstrated that CD9 decreased the phosphorylation of EGFR at specific sites. In addition, CD9 attenuated EGFR signaling of PI3K/Akt and MAPK/Erk, which was associated with cell growth and proliferation. Conversely, small hairpin RNA-mediated knockdown of CD9 expression enhanced the activation of EGFR signal transduction pathways, including PI3K/Akt and MAPK/Erk. These results suggested that the mechanism underlying CD9-induced suppression of cell proliferation may involve the inhibition of phosphorylation of EGFR and the activity of PI3K/Akt and MAPK/Erk signaling pathways.
\end{abstract}

\section{Introduction}

Glioblastoma multiforme (GBM) is the most common and aggressive form of brain cancer. GBM is a highly aggressive neuroepithelial tumor, which grows almost exclusively in neural tissue (1). The overall survival rate of patients with GBM has increased in the last decade due to the use of

Correspondence to: Mr. Gong-Ping Wang, First Department of Neurosurgery, Xianyang Hospital of Yanan University, Central Hospital of 20th Bureau of China Railway Group, 38 Wenlin Road, Xianyang, Shaanxi 712000, P.R. China

E-mail: dr_gongping@sina.com

Key words: CD9, proliferation, epidermal growth factor receptor, phosphoinositide-3-kinase, mitogen-activated protein kinase, phosphorylation aggressive surgery combined with radiation, chemotherapy or biological therapy. However, malignant glioma cell proliferation and invasion remain the predominant factors affecting patient mortality $(2,3)$. At the time of GBM diagnosis, in the majority of cases, the percentage of patients with a two-year survival rate is $<30 \%$ (4). This severely malignant phenotype is at least partially due to acquired excess cell proliferation. A recent development in cancer research is the discovery of the tetraspanin proteins, which act as molecular facilitators of adaptors to organize a network of interactions among the cell surface molecules, known as the 'tetraspanin web'. Members of the tetraspanin protein family are involved in regulation of the cell cycle as well as cell growth and proliferation. The mechanisms underlying the growth and proliferation of GBM cells remain to be elucidated; therefore, further study is required to improve therapeutic strategies.

CD9 is a cell surface glycoprotein of the tetraspanin protein family, which has a characteristic structure of four transmembrane domains and two extracellular loops. Tetraspanins can interact with other functional proteins, including growth factor receptors, intracellular signaling molecules and integrins, to form multiprotein complexes at the tetraspanin-enriched microdomain at the cell surface (5). Tetraspanins are involved in various biological processes, including cell survival, growth and migration. The tetraspanin CD9 was initially identified as a metastasis suppressor of solid tumors and was shown to be expressed in numerous types of cell, including platelets, pre-B cells and Schwann cells. Clinical and pathological findings have demonstrated that reduced CD9 gene expression is associated with poor prognoses in breast $(6,7)$, colon (8) and lung cancer (9). Recent studies have suggested that CD9 may have the potential to regulate motility, through other transmembrane proteins $(10,11)$. Furthermore, it has previously been reported that CD9 is directly associated with epidermal growth factor receptor (EGFR) and is able to destabilize surface expression of EGFR and consequently attenuate ligand-induced activation of the receptor (12).

A common characteristic of GBM histology is EGFR amplification, which affects signal transduction processes $(13,14)$. EGFR signaling occurs through a complex network of intermediates, including phosphoinositide-3-kinase (PI3K), mitogen-activated protein kinase (MAPK) and phospholipase $\mathrm{C}-\gamma$ (15). The dysregulation of signal transduction processes affects various downstream biological processes 
associated with cell proliferation, migration, adhesion and invasion $(16,17)$. Nakamura et al (18) previously reported that CD9 affects EGFR ligand binding activities of the membrane-bound forms of transforming growth factor- $\alpha$ and heparin-binding EGF-like growth factor, which may contribute to human malignant glioma cell growth.

The present study aimed to present a novel mechanistic insight into the important role of tetraspanin CD9 in regulating the proliferation of human glioblastoma cells through epidermal growth factor receptor (EGFR) activity. The regulation of the expression and phosphorylation of EGFR and EGFR-induced signaling were examined in order to determined whether the function of CD9 was linked the inhibition of proliferation in human glioblastoma cells.

\section{Materials and methods}

Cell line and transfection. The LN229 human glioblastoma cells were obtained from the American Type Culture Collection (Manassas, VA, USA). The cells were cultured in Dulbecco's modified Eagle's medium supplemented with $10 \%$ fetal bovine serum (Gibco-BRL, Carlsbad, CA, USA), $50 \mathrm{mg} / \mathrm{ml}$ streptomycin and $50 \mathrm{U} / \mathrm{ml}$ penicillin (Gibco-BRL), in a humidified atmosphere containing $5 \% \mathrm{CO}_{2}$ at $37^{\circ} \mathrm{C}$. The $\mathrm{LN} 229$ cells were stably transfected with a CD9/pcDNA3.1 plasmid (LN/CD9) or a control pcDNA3.1 plasmid (LN/cont). The coding region of CD9 cDNA was generated through reverse transcription quantitative-polymerase chain reaction (RT-qPCR) from LN229 human glioblastoma cells. The CD9 cDNA fragments were amplified by RT-qPCR, were digested with $B a m \mathrm{HI}$ and $E c o$ RI, and the purified cDNA fragments were ligated into the BamHI-EcoRI-digested pcDNA3.1 (Invitrogen Life Technologies, Carlsbad, CA, USA). The primers used in the PCR were as follows: forward, 5'-CGGGATCCACCATGCCGGTCAAAGGAGGCA-3' and reverse,5'-CGGAATTCCGCTAGACCATCTCGCGGTTCC-3' (Takara Biotechnology Co., Ltd., Dalian, China). The construct was verified by sequencing. LN229 cell lines were transfected with a CD9/pcDNA 3.1 using the Lipofectamine 2000 reagent (Invitrogen Life Technologies) followed by selection in Zeocin (Invitrogen Life Technologies). A CD9 small hairpin (sh)RNA plasmid (LN/shCD9) was constructed in order to generate CD9-depleted cells. The cells transfected with a negative control (NC) shRNA plasmid were considered control cells (shNC) and untransfected LN229 cells were considered the mock control cells. A CD9 shRNA and a negative control plasmid were constructed by GenePharma using pGPH1/Neo plasmid (GenePharma, Shanghai, China) followed by selection in G418 (Gibco BRL). The 21 nt siRNA directed against CD9 was 5'-UUCUUGCUCGAAGAUGCUCTT-3'. LN229 cells were transfected using Lipofectamine ${ }^{\circledR} 2000$ reagent (Invitrogen Life Technologies), according to the manufacturer's instructions.

Antibodies and reagents. Primary antibodies against the following antigens were all purchased from Santa Cruz Biotechnology, Inc. (Dallas, TX, USA): Rabbit polyclonal CD9 immunoglobulin (Ig)G (1:500; sc9148), goat polyclonal EGFR IgG (1:1,000; sc31157), goat polyclonal p-EGFR (Tyr-1173) IgG (1:500; sc12351), mouse monoclonal p-EGFR (Tyr-1086) IgG (1:500; sc81490), rabbit polyclonal p-Akt1/2/3 (Ser473) IgG (1:1,000; sc33437), rabbit polyclonal p-Akt1/2/3
A

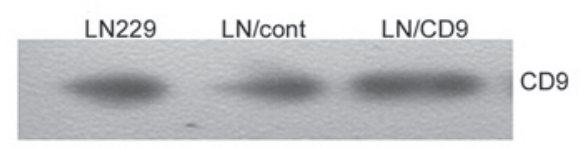

B
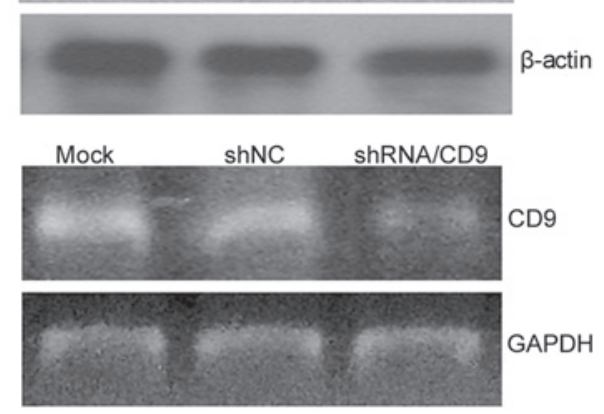

C
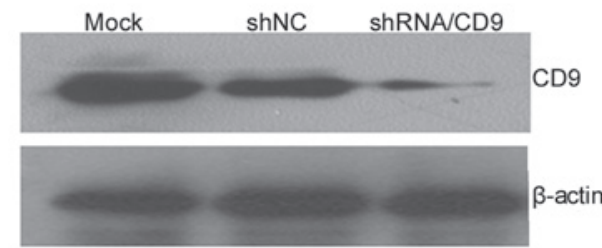

Figure 1. Modulating CD9 expression in LN229 human glioblastoma cells. Cells were seeded into 12-well plates in Dulbecco's modified Eagle's medium supplemented with $10 \%$ FBS. LN229 cells were transfected with a CD9/pcDNA3.1 plasmid or a CD9 shRNA plasmid. (A) Cells were measured for overexpression of CD9 following transfection with pcDNA3.1/CD9 plasmid (LN/CD9) or an empty pcDNA3.1 plasmid (LN/cont). Untransfected LN229 cells were used as a control. CD9 protein expression levels were measured by immunoblotting with a CD 9 antibody, and $\beta$-actin antibody was used as a loading control. Cells were also transfected with CD9 shRNA plasmid (LN/shCD9) or a shNC plasmid. Untransfected LN229 cells (mock) were used as a control. The mRNA and protein expression levels of CD9 were determined by (A and C) western blot analysis and (B) reverse transcription polymerase chain reaction, respectively. shRNA, small hairpin RNA; NC, negative control.

(Thr308) IgG (1:1,000; sc135650), rabbit polyclonal Akt1/2/3 $\operatorname{IgG}(1: 1,000 ;$ sc8312), goatpolyclonalp-Erk1/2(Thr202/Tyr204) IgG (1:1,000; sc16982), rabbit polyclonal Erk1/2 IgG (1:1,000; sc292838), goat polyclonal $\beta$-actin $\operatorname{IgG}(1: 1,000$; sc1616). The PVDF membranes were incubated with the primary antibodies overnight at $4^{\circ} \mathrm{C}$. Secondary antibodies $(1: 2,000)$, including goat anti-rabbit horseradish peroxidase (HRP)-conjugated $\mathrm{IgG}$, rabbit anti-goat HRP-conjugated IgG and goat anti-mouse HRP-conjugated IgG-horseradish peroxidase (Zhongshan Goldenbridge Biotechnology Co., Beijing, China), for $1 \mathrm{~h}$ at room temperature.

EGF was obtained from ProSpec-Tany TechnoGene Ltd. (Rehovot, Israel). Akt inhibitor LY294002 and Erk inhibitor U0126 were purchased from Abcam (Hong Kong, China). Opti-MEM was obtained from Invitrogen Life Technologies. Protease inhibitor cocktail was obtained from Sigma-Aldrich (St. Louis, MO, USA).

$R T$-PCR. Total RNA was extracted from the cells using TRIzol $^{\circledR}$ (Invitrogen Life Technologies), and $1 \mu \mathrm{g}$ total RNA was reverse-transcribed using a cDNA Synthesis kit (Invitrogen Life Technologies) with random hexamers. The PCR cycling parameters were set as follows: 30 cycles of $40 \mathrm{sec}$ at $94^{\circ} \mathrm{C}$, $40 \mathrm{sec}$ at $60^{\circ} \mathrm{C}$ and $60 \mathrm{sec}$ at $72^{\circ} \mathrm{C}$. The identity of the PCR products was confirmed through sequencing by BGI Tech Solutions Co. (Shenzhen, China) using T7 primer. The relative quantitative analysis was normalized to the endogenous 
A

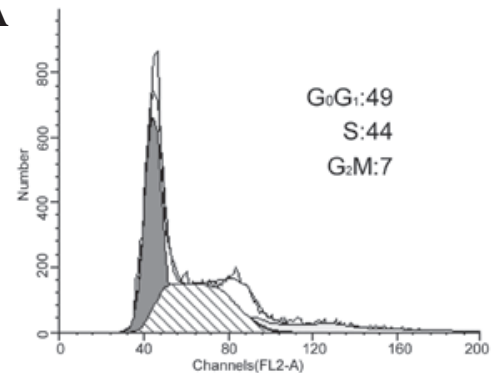

C

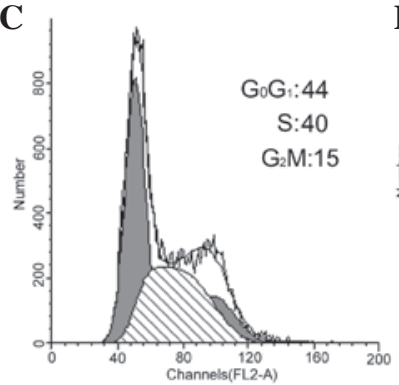

B

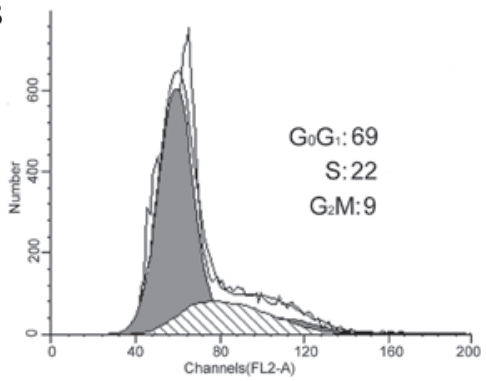

E

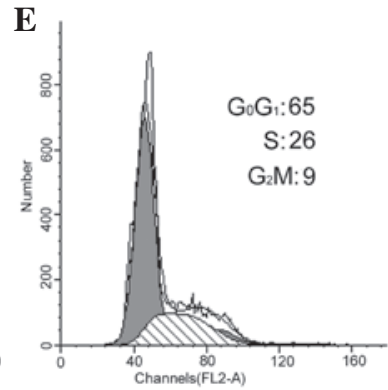

Figure 2. Effects of CD9 and inhibition of the PI3K/Akt and MAPK/Erk signaling pathways using LY294002 and U0126, respectively, on the proliferation of LN229 human glioblastoma cells stimulated with EGF, using flow cytometry. Cells transfected with CD9/pcDNA3.1 plasmid or an empty pcDNA3.1 plasmid were serum-starved for $12 \mathrm{~h}$ in the presence of the desired regents and then stimulated with $50 \mathrm{ng} / \mathrm{ml}$ EGF. (A and B) Flow cytometry was performed to measure the proliferation efficiency of CD9 in LN229 cells transfected with a control pcDNA3.1 plasmid (LN/cont) or a CD9/pcDNA3.1 plasmid (LN/CD9). (C-E) Treated cells were serum-starved for $12 \mathrm{~h}$ in the presence of the desired regents and the PI3K/Akt and MAPK/Erk signaling pathways were inhibited in the cells using $15 \mu \mathrm{M}$ LY294002 or $20 \mu \mathrm{M}$ U0126 for $6 \mathrm{~h}$, respectively. The proliferation efficiency of the cells stimulated with $50 \mathrm{ng} / \mathrm{ml}$ EGF was measured as described in the Materials and Methods. (A) +EGF+pcDNA3.1 (control), (B) +EGF,+CD9/pcDNA3.1, (C) +EGF (control), (D) +EGF+LY294002, (E) +EGF+U0126. MAPK, mitogen-activated protein kinase; PI3K, phosphoinositide-3-kinase; Erk, extracellular signal-regulated kinase; EGF, epidermal growth factor.

control GAPDH. The following primer sequences were used: GAPDH forward, TACTTATGCCGATGTCGTTGT and reverse, CCAGCCTCGTCCCGTAGA; CD9 forward, TGC ATCTGTATCCAGCGCCA and reverse, CTCAGGGATG TAAGCTGACT (Takara Biotechnology Co., Inc.) (19).

Flow cytometry. Cell cycle distribution was analyzed using flow cytometry with propidium iodide (PI) staining. Briefly, $1.5 \times 10^{5}$ cells transfected with CD9 and negative control plasmids for four days were seeded in 6-cm dishes and cultured for two days at $37^{\circ} \mathrm{C}$. The cells were then treated with the desired agents as described in the figure legends, in serum-free medium overnight. The medium was refreshed and the cells were stimulated with $50 \mathrm{ng} / \mathrm{ml}$ EGF for $10 \mathrm{~min}$ at room temperature. Cells $\left(1 \times 10^{4}\right)$ were then harvested by adding $0.05 \%$ trypsin $/ 0.04 \%$ EDTA and washed twice with PBS. The cells were subsequently fixed in ice cold $70 \%$ ethanol for $1 \mathrm{~h}$ and treated with RNAase and PBS, containing $100 \mu \mathrm{g} / \mathrm{ml}$ DNase-free RNase and $40 \mu \mathrm{g} / \mathrm{ml}$ PI (Sigma-Aldrich), then incubated for $1 \mathrm{~h}$ at $37^{\circ} \mathrm{C}$. A total of 10,000 stained nuclei were analyzed using a FACSCalibur flow cytometer (BD Biosciences, Franklin Lakes, NJ, USA). To identify the effects of the PI3K/Akt and MAPK/Erk pathways on the regulation of cell proliferation, these two signaling pathway were inhibited using $15 \mu \mathrm{M}$ LY294002 or $20 \mu \mathrm{M}$ U0126 for $6 \mathrm{~h}$, respectively. The cells were then subjected to flow cytometry, as described above.

Western blot analysis. Cells $\left(1 \times 10^{5}\right)$ were treated as described above and seeded in 12-well plates (Falcon; Fisher Thermo Scientific, Waltham, MA, USA). The cells were incubated overnight in serum-free medium. The medium was replaced and the cells were stimulated with $50 \mathrm{ng} / \mathrm{ml} \mathrm{EGF}$ for $10 \mathrm{~min}$ at room temperature. The cells were then harvested and lysed in $200 \mu 1$ radioimmunoprecipitation lysis buffer $(50 \mathrm{mM}$ Tris, $\mathrm{pH} 7.5,150 \mathrm{mM} \mathrm{NaCl}, 1 \%$ Triton X-100, $25 \mathrm{mM} 0.5 \%$ sodium deoxycholate, $0.1 \%$ SDS, $5 \mathrm{mM}$ pyrophosphate and $50 \mathrm{mM}$ NaF; Sigma-Aldrich), supplemented with $1 \mathrm{mM}$ $\mathrm{Na}_{3} \mathrm{VO}_{4}, 1 \mathrm{mM}$ dithiothreitol, $1 \%$ protease inhibitor cocktail and $1 \%$ phosphatase inhibitor cocktail per well. $10 \mu \mathrm{l}$ inhibitor cocktail (Sigma-Aldrich) was added to $1 \mathrm{ml}$ lysis buffer, all reagents described above were purchased from Sangon Biotech Technogene Ltd. (Shanghai, China). The lysate was determined using a bicinchoninic acid protein assay kit (Beyotime, Jiangsu, China). Heat-denatured protein samples (20 $\mu \mathrm{g}$ per lane) were separated using 12\% SDS-PAGE (Invitrogen Life Technologies) and the proteins were transferred to polyvinylidene fluoride (PVDF) membranes (Millipore, Bedford, MA, USA). The non-specific binding sites on the PVDF membranes were blocked with $5 \%$ nonfat dry milk in tris-buffered saline with $0.1 \%$ Tween-20. The target protein bands in the PVDF membranes were revealed by immunoblotting with primary antibodies, followed by an incubation with the species-specific HRP-conjugated secondary antibodies. Signal bands were detected using enhanced chemiluminescence reagents (GE Healthcare Life Sciences, Little Chalfont, UK). $\beta$-actin was used as a housekeeping antibody, to verify equal amounts of protein were loaded in all of the lanes.

Statistical analysis. All of the experiments were repeated at least three times and consistently yielded similar results. The data were analyzed by GraphPad Prism version 5.0 (GraphPad Software Inc., La Jolla, CA, USA). Statistical comparisons between groups were performed using a one-way analysis of variance. $\mathrm{P}<0.01$ was considered to indicate a statistically 
A

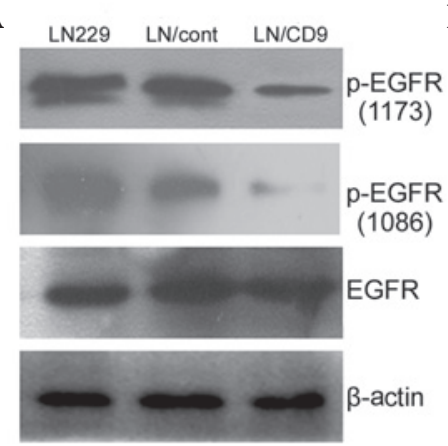

B

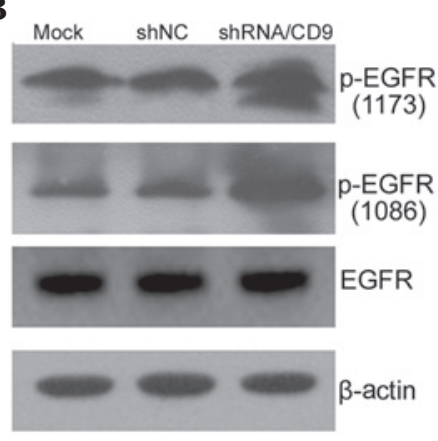

Figure 3. Effect of CD9 on the phosphorylation and expression of EGFR in LN229 human glioblastoma cells. Treated cells were serum-starved for $12 \mathrm{~h}$ and then stimulated with $50 \mathrm{ng} / \mathrm{ml} \mathrm{EGF}$ for $10 \mathrm{~min}$. The cells were then harvested and subjected to western blot analysis with antibodies targeting p-EGFR (Tyr-1173), p-EGFR (Tyr-1086) and EGFR. (A) Increased CD9 content inhibited EGF-stimulated phosphorylation of EGFR at Tyr-1173 and Tyr-1086. (B) Decreased CD9 content upregulated EGF-stimulated phosphorylation of EGFR at Tyr-1173 and Tyr-1086. All experiments were performed at least three times. EGFR, epidermal growth factor receptor; p, phosphorylated; shRNA, small hairpin RNA; cont, control; NC, negative control.

A

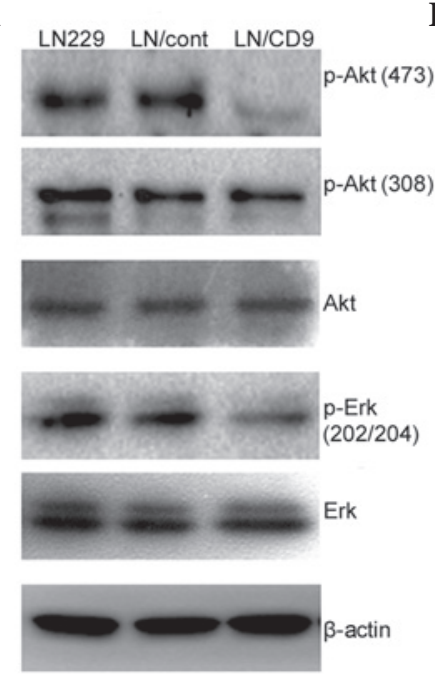

B

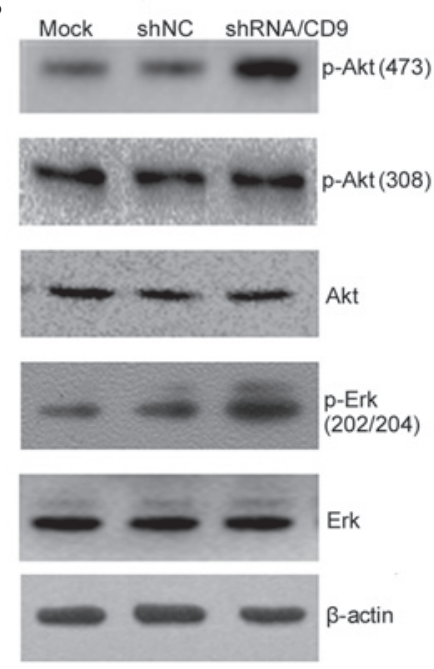

Figure 4. Effects of CD9 on the PI3K/Akt and MAPK/Erk signaling pathways in LN229 human glioblastoma cells. Treated cells were serum-starved for $12 \mathrm{~h}$ in the presence of the desired regents and then stimulated with $50 \mathrm{ng} / \mathrm{ml}$ EGF for $10 \mathrm{~min}$. The cells were then harvested and subjected to western blot analysis with antibodies targeting Akt 1/2/3, p-Akt1/2/3 (Thr308), p-Akt 1/2/3 (Ser473), p-p44/42 MAPK (Thr202/Tyr204) and Erk1/2 . (A) Increased CD9 content inhibited EGF-stimulated phosphorylation of Akt at Ser473 and Erk at Erk1 and 2. (B) Decreased CD9 content upregulated EGF-stimulated phosphorylation of Akt at Ser473 and Erk at Erk1 and 2. All experiments were performed at least three times. MAPK, mitogen-activated protein kinase; PI3K, phosphoinositide-3-kinase; Erk, extracellular signal-regulated protein kinase; EGF, epidermal growth factor; p, phosphorylated; shRNA, small hairpin RNA; $\mathrm{NC}$, negative control.

significant difference between values. Values are expressed as the mean \pm standard error of the mean.

\section{Results}

CD9 expression in LN229 cells. The CD9 gene was constructed into a pcDNA3.1 vector and transfected into LN229 cells (LN/CD9). The protein expression levels of CD9 were measured after 48 hours by western blotting. The protein expression levels of CD9 were significantly enhanced in the LN229 cells transfected with the pcDNA 3.1/CD9 vector, as compared with the cells transfected with an empty pcDNA3.1 vector (Fig. 1A). The LN229 cells were also transfected with CD9 shRNA plasmids in order to suppress CD9 expression (LN/shCD9). RT-PCR and western blot analyses indicated that the expression levels of endogenous CD9 were stably decreased by $\sim 70 \%$ (Fig. 1B and C).
Overexpression of CD9 inhibits EGF-stimulated LN229 cell proliferation EGF and affects the phosphorylation of EGFR. To elucidate whether CD9 is able to inhibit cell growth by affecting cell cycle progression, the cell cycle distribution was assessed in the LN229 cells by flow cytometry. The cell population in the LN/CD9 group displayed a significant increase in the proportion of cells in $\mathrm{G}_{0} / \mathrm{G}_{1}$ phase, and a significant decrease in the number of cells in the $\mathrm{S}$ phase, as compared with the control cells (Fig. 2A and B). These results suggested that CD9 may inhibit the proliferation of LN229 cells via cell cycle regulation. To investigate the molecular mechanisms underlying how CD9 affects EGF-stimulated cell growth, the expression and phosphorylation status of EGFR was determined by western blotting. Increased CD9 expression significantly reduced EGF-stimulated phosphorylation of EGFR at Y1173 and Y1086 without significantly affecting EGFR expression levels (Fig. 3A), while downregulation of 
CD9 elevated EGF-stimulated phosphorylation of EGFR at Y1173 and Y1086 (Fig. 3B).

Effects of inhibiting the PI3K/Akt and MAPK/Erk pathways. To investigate how CD9 modulated LN229 cell proliferation and growth regulation via the PI3K/Akt and MAPK/Erk pathways, the EGF-stimulated LN229 cells were transfected with an empty pcDNA3.1 vector and were treated with LY294002 and U0126, respectively. Inhibition of the PI3K/Akt and MAPK/Erk pathways, using LY294002 and U0126, suppressed EGF-stimulated proliferation (Fig. 2C-E). These results suggested that the PI3K/Akt and MAPK/Erk pathways may be involved in the modulation of LN229 cell growth regulation in vitro.

CD9 attenuates EGFR signaling of PI3K/Akt and MAPK/Erk pathways. Based on previous results, it was hypothesized that PI3K/Akt and MAPK/Erk pathways may be associated with glioma cell proliferation. To further investigate the mechanisms underlying the suppressive effects of CD9 on EGF-stimulated growth in vitro, these two signaling pathways, which are essential for the modulation of tumor cell growth, were measured by western blot analysis. Overexpression or suppression of CD9 directly affected the activation of the PI3K/Akt and MAPK/Erk pathways. CD9 negatively affected EGFR-mediated activation of Akt and Erk (Fig. 4). In the EGF-treated LN229 cells overexpressing CD9, the inhibitory effect of CD9 on phosphorylation of Akt at Ser473 was enhanced, and the opposite effect was observed on the phosphorylation of Akt at Ser473 and Erk at Erk1 and 2 following knockdown of CD9 (Fig. 4A and B). However, alterations in the cellular content of CD9 did not affect the level of EGF-induced phosphorylation of Akt at Thr308. These results indicated that the PI3K/Akt and MAPK/Erk signaling pathways may have an important role in CD9-regulated cell proliferation.

\section{Discussion}

The present study demonstrated that CD9 decreased the phosphorylation of EGFR at specific sites. CD9 attenuated EGFR signaling of PI3K/Akt and MAPK/Erk, which are associated with cell growth and proliferation. Conversely, shRNA-mediated knockdown of CD9 expression enhanced the activation of the EGFR signal transduction pathway, including PI3K/Akt and MAPK/Erk, which enhanced cell proliferation. This activation was blocked by treatment with PI3K and MAPK inhibitors.

The tetraspanin CD9 was initially characterized as a cell surface antigen on lymphohemopoietic cells, platelets, eosinophil and pre-B cells $(20,21)$. CD9 is also normally expressed in mature oligodendrocytes and Schwann cells, and is implicated in neurite outgrowth and myelination $(22,23)$. Recently, numerous clinical studies have demonstrated that CD9 expression is inversely correlated with patient survival and metastatic potential in various types of human cancer $(6-9,24)$. Conversely, Kawashima et al (25) demonstrated that among the neuroepithelial tumors; high-grade astrocytic tumors, including glioblastomas, had higher immunoreactivity of CD9, as compared with low-grade cerebral astrocytomas, thus suggesting that CD9 expression in astrocytic tumors may be correlated with their malignancy (25). Tetraspanin members, such as CD82, can associate with EGFR and attenuate EGFR signaling by accelerating EGFR internalization, and redistribute EGFR into distinct cellular compartments following endocytosis. This process significantly impacts the early events associated with receptor activation, including ligand binding, dimerization and re-localization of activated receptors into clathrin-coated pits $(26,27)$. For numerous reasons, the present study hypothesized that CD9 may affect glioma tumor biology. To fully understand the roles of CD9 on glioma tumor biology, CD9 expression was modulated in the LN229 human glioblastoma cell line, and it was determined whether CD9 was able to drive cell proliferation. Numerous studies have suggested that CD9 provides a bridge among cell surface proteins, including growth factor receptors, to generate functional complexes involved in cell proliferation, division migration and apoptosis $(28,29)$. To explore the precise roles and mechanistic action of CD9 on the proliferation of glioblastomas, the present study focused on the association between CD9 and EGFR, and the EGFR signals mediated by CD9 (30). CD9 was shown to decrease the phosphorylation of particular tyrosine residues and reduce the EGFR signals, which usually mediate the proliferation and survival of glioblastoma.

The EGFR is the prototypical member of the ErbB/EGFR family, which is a primary contributor to glioblastoma initiation and progression. To clarify the role of the association between EGFR and CD9, CD9 content was altered in LN229 cells by transfection with CD9 and shRNA-CD9. The results demonstrated that CD9 inhibited EGF-stimulated phosphorylation at Y1173 and Y1086. EGFR phosphorylation leads to the recruitment of complex effector proteins through recognition and binding of Src homology 2 and phosphotyrosine binding domains on the effector proteins, to phosphotyrosine motifs on the receptor (31-33). Consequently, various downstream signaling cascades, including the PI3K and MAPK are activated.

The results of the present study demonstrated that inhibitors of PI3K or MAPK blocked the proliferation of LN229 cells, which indicates that the PI3K/Akt and MAPK/Erk pathways are important for cell growth and survival $(34,35)$. The effects of CD9 were then determined on the activity of these two signaling pathways stimulated by EGF. The results suggested that CD9 negatively regulated EGF-stimulated activation of PI3K/Akt phosphorylation at Ser473 and MAPK phosphorylation, but did not affect PI3K/Akt phosphorylation at Thr308. Flow cytometric analyses confirmed that CD9 had negligible effects on EGF-stimulated cell proliferation. Phosphorylation of PI3K/Akt by phosphoinositide-dependent protein kinase 1 at Thr308 and at Ser473 by the mammalian target of rapamycin complex 2 is required for full kinase activity (36). The results of the present study suggested that Akt Ser473 phosphorylation contributes to CD9-mediated cell proliferation in response to EGF. In addition, CD9 affects the MAPK/Erk signaling pathway. Activation of the MAPK/Erk pathway is triggered by growth factor receptor-bound protein 2 binding directly to the receptor at Y1068, and indirectly through Src homology domain-containing adaptor protein $\mathrm{C}$ binding at Y1173 and Y1148 (37). Initial experiments demonstrated that CD9 suppressed EGF-stimulated EGFR phosphorylation at Y1173 
and Y1086. Therefore, it may be hypothesized that CD9 can regulate the phosphorylation of Y1173 and Y1086 residues on EGFR, which is responsible for the EGF-induced activation of PI3K/Akt and MAPK/Erk. The changes to the levels of phosphorylation at the Y1173 and Y1086 residues were consistent with those of the cellular content of CD9.

In conclusion, upregulated CD9 expression in LN229 cells inhibited cell proliferation, decreased EGF-induced phosphorylation of PI3K/Akt at Ser473 and Erk1/2, and reduced EGF-induced phosphorylation at Y1173 and Y1086 residues on EGFR. Conversely, knockdown of CD9 expression resulted in attenuation of EGF-induced activation of PI3K/Akt and MAPK/Erk, and enhanced the EGF-induced phosphorylation of EGFR at Y1173 and Y1086. These results suggested that the mechanism by which CD9 affects EGFR signaling appears rather complex and requires further study.

\section{References}

1. Thorsen F and Tysnes BB: Brain tumor cell invasion, anatomical and biological considerations. Anticancer Res 17: 4121-4126, 1997.

2. Ohgaki $\mathrm{H}$ and Kleihues P: Epidemiology and etiology of gliomas. Acta Neuropathol 109: 93-108, 2005.

3. Parsons DW, Jones S, Zhang X, et al: An integrated genomic analysis of human glioblastoma multiforme. Science 321: 1807-1812, 2008.

4. Stupp R, Mason WP, van den Bent MJ, et al; European Organisation for Research and Treatment of Cancer Brain Tumor and Radiotherapy Groups; National Cancer Institute of Canada Clinical Trials Group: Radiotherapy plus concomitant and adjuvant temozolomide for glioblastoma. N Engl J Med 352: 987-996, 2005

5. Regina Todeschini A and Hakomori SI: Functional role of glycosphingolipids and gangliosides in control of cell adhesion, motility, and growth, through glycosynaptic microdomains. Biochim Biophys Acta 1780: 421-433, 2008.

6. Miyake M, Nakano K, Itoi SI, Koh T and Taki T: Motility-related protein-1 (MRP-1/CD9) reduction as a factor of poor prognosis in breast cancer. Cancer Res 56: 1244-1249, 1996.

7. Huang CI, Kohno N, Ogawa E, Adachi M, Taki T and Miyake M: Correlation of reduction in MRP-1/CD9 and KAI1/CD82 expression with recurrences in breast cancer patients. Am J Pathol 153: 973-983, 1998.

8. Mori M, Mimori K, Shiraishi T, et al: Motility related protein 1 (MRP1/CD9) expression in colon cancer. Clin Cancer Res 4: $1507-1510,1998$.

9. Higashiyama M,Doi O,Kodama K, et al: Immunohistochemically detected expression of motility-related protein-1 (MRP-1/CD9) in lung adenocarcinoma and its relation to prognosis. Int J Cancer 74 : 205-211, 1997.

10. Halova I, Dráberová L, Bambousková M, et al: Cross-talk between tetraspanin CD9 and transmembrane adaptor protein non-T cell activation linker (NTAL) in mast cell activation and chemotaxis. J Biol Chem 288: 9801-9814, 2013.

11. Huang CL, Liu D, Masuya D, et al: MRP-1/CD9 gene transduction downregulates Wnt signal pathways. Oncogene 23: 7475-7483, 2004.

12. Murayama Y, Shinomura Y, Oritani K, et al: The tetraspanin CD9 modulates epidermal growth factor receptor signaling in cancer cells. J Cell Physiol 216: 135-143, 2008.

13. Liu L, Bäcklund LM, Nilsson BR, et al: Clinical significance of EGFR amplification and the aberrant EGFRvIII transcript in conventionally treated astrocytic gliomas. J Mol Med (Berl) 83: 917-926, 2005

14. Sugawa N, Yamamoto K, Ueda S, et al: Function of aberrant EGFR in malignant gliomas. Brain Tumor Pathol 15: 53-57, 1998

15. Wang HX, Li Q, Sharma C, Knoblich K and Hemler ME: Tetraspanin protein contributions to cancer. Biochem Soc Trans 39: 547-552, 20w11.

16. Huang X, Li Y, Zhang J, Xu Y, Tian Y and Ma K: Ganglioside GM3 inhibits hepatoma cell motility via down-regulating activity of EGFR and PI3K/AKT signaling pathway. J Cell Biochem 114: 1616-1624, 2013
17. Li Y, Huang X, Zhong W, Zhang J and Ma K: Ganglioside GM3 promotes HGF-stimulated motility of murine hepatoma cell through enhanced phosphorylation of cMet at specific tyrosine sites and PI3K/Akt-mediated migration signaling. Mol Cell Biochem 382: 83-92, 2013.

18. Nakamura K, Mitamura T, Takahashi T, Kobayashi T and Mekada E: Importance of the major extracellular domain of CD9 and the epidermal growth factor (EGF)-like domain of heparin-binding EGF-like growth factor for up-regulation of binding and activity. J Biol Chem 275: 18284-18290, 2000.

19. Funakoshi T, Tachibana I, Hoshida Y, et al: Expression of tetraspanins in human lung cancer cells: frequent downregulation of CD9 and its contribution to cell motility in small cell lung cancer. Oncogene 22: 674-687, 2003.

20. Boucheix C and Benoit P: CD9 antigen: will platelet physiology help to explain the function of a surface molecule during hemopoietic differentiation? Nouv Rev Fr Hematol 30: 201-202, 1988.

21. Masellis-Smith A and Shaw AR: CD9-regulated adhesion. Anti-CD9 monoclonal antibody induce pre-B cell adhesion to bone marrow fibroblasts through de novo recognition of fibronectin. J Immunol 152: 2768-2777, 1994.

22. Schmidt C, Künemund V, Wintergerst ES, Schmitz B and Schachner M: CD9 of mouse brain is implicated in neurite outgrowth and cell migration in vitro and is associated with the alpha 6/beta 1 integrin and the neural adhesion molecule L1. J Neurosci Res 43: 12-31, 1996.

23. Chernousov MA, Stahl RC and Carey DJ: Tetraspanins are involved in Schwann cell-axon interaction. J Neurosci Res 91: 1419-1428, 2013.

24. Murayama Y, Miyagawa J, Shinomura Y, et al: Significance of the association between heparin-binding epidermal growth factor-like growth factor and CD9 in human gastric cancer. Int J Cancer 98: 505-513, 2002.

25. Kawashima M, Doh-ura K, Mekada E, Fukui M and Iwaki T: CD9 expression in solid non-neuroepithelial tumors and infiltrative astrocytic tumors. J Histochem Cytochem 50: 1195-1203, 2002.

26. Takahashi M, Sugiura T, Abe M, Ishii K and Shirasuna K: Regulation of c-Met signaling by the tetraspanin KAI-1/CD82 affects cancer cell migration. Int J Cancer 121: 1919-1929, 2007.

27. Odintsova E, Voortman J, Gilbert E and Berditchevski F Tetraspanin CD82 regulates compartmentalisation and ligand-induced dimerization of EGFR. J Cell Sci 116: 4557-4566, 2003.

28. Gustafson-Wagner E and Stipp CS: The CD9/CD81 tetraspanin complex and tetraspanin CD151 regulate alpha3beta1 integrin-dependent tumor cell behaviors by overlapping but distinct mechanisms. PloS One 8: e61834, 2013.

29. Nakagawa T, Higashiyama S, Mitamura T, Mekada E and Taniguchi N: Amino-terminal processing of cell surface heparin-binding epidermal growth factor-like growth factor up-regulates its juxtacrine but not its paracrine growth factor activity. J Biol Chem 271: 30858-30863, 1996.

30. Murayama Y, Miyagawa J, Oritani K, et al: CD9-mediated activation of the $\mathrm{p} 46 \mathrm{Shc}$ isoform leads to apoptosis in cancer cells. J Cell Sci 117 (Pt 15): 3379-3388, 2004.

31. Jones RB, Gordus A, Krall JA and MacBeath G: A quantitative protein interaction network for the ErbB receptors using protein microarrays. Nature 439: 168-174, 2006.

32. Lindemann C, Hackmann O, Delic S, Schmidt N, Reifenberger G and Riemenschneider MJ: SOCS3 promoter methylation is mutually exclusive to EGFR amplification in gliomas and promotes glioma cell invasion through STAT3 and FAK activation. Acta Neuropathol 122: 241-251, 2011.

33. Jin W, Xu X, Yang T and Hua Z: p53 mutation, EGFR gene amplification and loss of heterozygosity on chromosome 10,17 p in human gliomas. Chin Med J (Engl) 113: 662-666, 2000.

34. Cecconi S, Mauro A, Cellini V and Patacchiola F: The role of Akt signalling in the mammalian ovary. Int J Dev Biol 56: 809-817, 2012.

35. Golding SE, Morgan RN, Adams BR, Hawkins AJ, Povirk LF and Valerie K: Pro-survival AKT and ERK signaling from EGFR and mutant EGFRvIII enhances DNA double-strand break repair in human glioma cells. Cancer Biol Ther 8: 730-738, 2009.

36. Sarbassov DD, Guertin DA, Ali SM and Sabatini DM: Phosphorylation and regulation of Akt/PKB by the rictor-mTOR complex. Science 307: 1098-1101, 2005

37. Schulze WX, Deng L and Mann M: Phosphotyrosine interactome of the ErbB-receptor kinase family. Mol Syst Biol 1: 2005.0008, 2005. 